\title{
Breastfeeding and Childhood Dental Caries: Results from a Socially Diverse Birth Cohort Study
}

\author{
Agatha W. van Meijeren-van Lunteren ${ }^{a, b}$ Trudy Voortman ${ }^{a, c}$ \\ Marlies E.C. Elfrink ${ }^{a, d}$ Eppo B. Wolvius ${ }^{a, b}$ Lea Kragt ${ }^{a, b}$ \\ aThe Generation R Study Group, Erasmus University Medical Centre, Rotterdam, The Netherlands; \\ ${ }^{b}$ Department of Oral and Maxillofacial Surgery, Special Dental Care and Orthodontics, Erasmus University Medical \\ Centre, Rotterdam, The Netherlands; ' Department of Epidemiology, Erasmus University Medical Centre, Rotterdam, \\ The Netherlands; ${ }^{d}$ Mondzorgcentrum Nijverdal, Nijverdal, The Netherlands
}

\section{Keywords}

Dental caries · Pediatric dentistry · Infant feeding ·

Breastfeeding $\cdot$ Nutritional epidemiology $\cdot$ Infants

\begin{abstract}
Previous studies showed that prolonged breastfeeding increases the risk of caries. However, the observed associations were mainly based on non-European populations, and important confounding and mediating factors like socioeconomic position (SEP) and diet were often neglected. The aim of this study was to investigate the role of breastfeeding and bottle-feeding practices on dental caries during childhood while accounting for SEP, ethnic background, and sugar intake. This study was part of the Generation R Study, a prospective multiethnic cohort study conducted in Rotterdam, The Netherlands. In total, 4,146 children were included in the analyses. Information about feeding practices was derived from delivery reports and questionnaires during infancy. Caries was measured via intraoral photographs at the age of 6 years and defined as decayed, missing, and filled teeth (dmft). Negative binomial hurdle regression analyses were used to study the associations between several infant feed-
\end{abstract}

ing practices and childhood caries. The prevalence of dental caries at the age of 6 years was $27.9 \%(n=1,158)$. Prolonged breastfeeding (for $>12$ months) was associated with dental caries (OR 1.35, 95\% $\mathrm{Cl} 1.04-1.74)$ and the number of teeth affected by dental caries (RR 1.27, 95\% Cl 1.03-1.56). Furthermore, nocturnal bottle-feeding was associated with dental caries (OR 1.52, 95\% Cl 1.20-1.93). All associations were independent of family SEP, ethnic background, and sugar intake. Results from this Dutch cohort study confirmed the previously observed associations between prolonged breastfeeding and nocturnal bottle-feeding and the increased risk of childhood dental caries, even after proper adjustments for indicators of SEP, ethnic background, and sugar intake. Future studies are encouraged to elaborate further on possible explanations for the observed relationships. Healthcare professionals should be aware and advise caregivers about the potential risk of prolonged breastfeeding on caries development by applying the current recommendations on breastfeeding, oral hygiene, and feeding frequency.
(C) 2021 The Author(s)

Published by S. Karger AG, Basel karger@karger.com www.karger.com/cre

Karger $\stackrel{\text { ' }}{5}$

GOPEN ACCESS
(C) 2021 The Author(s)

Published by S. Karger AG, Basel

This is an Open Access article licensed under the Creative Commons Attribution-NonCommercial-4.0 International License (CC BY-NC) (http://www.karger.com/Services/OpenAccessLicense), applicable to the online version of the article only. Usage and distribution for commercial purposes requires written permission.
Agatha W, van Meijeren-van Lunteren

The Generation R Study Group and Department of Oral and Maxillofacial Surgery Special Dental Care and Orthodontics, Erasmus University Medical Centre PO Box 2040, NL-3000 CA Rotterdam (The Netherlands)

a.vanlunteren@erasmusmc.nl 


\section{Introduction}

Dental caries is the most common chronic disease during childhood and has a negative impact on future dental and overall health [Kassebaum et al., 2015]. As a consequence, lifelong dental care can lead to high societal costs [Selwitz et al., 2007]. Moreover, especially children from families with a low socioeconomic position (SEP) are affected by poor oral health, including dental caries [Watt and Sheiham, 1999]. It is thus important to identify and understand the risk factors for dental caries in order to reduce oral health inequalities in the future.

The role of dietary carbohydrates on caries development is well-known, but the influence of infant feeding and its practices on childhood caries is poorly understood and can lead to confusion. The World Health Organization [2002] (WHO) recommends breastfeeding (exclusively) on demand within an hour after birth up to 6 months, and partial continuation of breastfeeding for up to 2 years, but in 2016 a review was published in the Lancet describing the lifelong effects of breastfeeding and showed that dental caries was the only negative health outcome related to prolonged breastfeeding [Victora et al., 2016]. Two other systematic reviews also found that prolonged breastfeeding increases the risk of childhood caries [Tham et al., 2015; Cui et al., 2017].

However, many of the previous studies did not take into account the role of family SEP, which is an important confounding factor affecting both infant feeding practices and dental caries. Moreover, few studies considered other nutritional practices, such as consumption of carbohydrate-containing foods, next to breastmilk or bottle-feeding. Therefore, it is still unclear whether dental caries is related to the carbohydrate content of breastmilk or coexisting factors. In addition, most of the previous studies were conducted in non-European countries, which makes it is difficult to generalize the results to a European population because the prevalence of caries and the oral health care system differ per country [Kassebaum et al., 2015; Lagerweij and van Loveren, 2015], and the presence of artificially fluoridated water could also have influenced previous study results [Ha et al., 2019].

The aim of this study was to investigate the role of breastfeeding and bottle-feeding practices on childhood dental caries independent of family SEP, ethnic background, and dietary habits, using data from a Dutch longitudinal multiethnic birth cohort study.

\section{Methods}

This study was conducted within the Generation R Study, an ongoing population-based prospective cohort study from fetal life onwards that was conducted in Rotterdam, The Netherlands. It is a multidisciplinary study focusing on several health outcomes from early life onwards. All pregnant mothers with an expected delivery date between April 2002 and January 2006 and who lived in city of Rotterdam were invited to participate in the Generation R study [Kooijman et al., 2016]. Based on the total number of live born children in the study area (Rotterdam), the response rate of the study at birth was estimated at $61 \%$ [Jaddoe et al., 2006]. The study population represented participants from different ethnic and socioeconomic groups [Kooijman et al., 2016].

Data collection started during pregnancy with physical assessments and through questionnaires. Prenatally, data collection continued, and is still ongoing at various time points through questionnaires, interviews, physical examination, behavior observations, biological sample collection, and MRI [Kooijman et al., 2016]. For our study, data collection took place during the preschool period at home-visits, repeated questionnaires, and routine child health center visits. Around the age of 6 years (between October 2008 and January 2012), 8,305 children (85\% of the original cohort $[n=9,749]$ ) participated in the Generation R study (Fig. 1) [Kooijman et al., 2016]. Of these, 2,978 had incomplete data on dental caries, and 1,181 had no data on $\geq 1$ of the infant feeding variables. Therefore, the total study population for the study consisted of 4,146 children with available data on $\geq 1$ feeding practices during infancy and caries assessment at the age of 6 years (Fig. 1).

Since 1976, drinking water has not been fluoridated in any part of The Netherlands.

\section{Assessment of Infant Feeding Practices}

Data on infant feeding were derived from delivery reports and questionnaires administered at the age of $2,6,12$, and 36 months [Durmuş et al., 2011]. Mothers were asked whether they ever breastfed their child (yes, no), whether they were still breastfeeding their child at the moment of filling in the questionnaire, and at what age they quit breastfeeding. Missing information on the initiation of breastfeeding from the questionnaires was complemented with data collected from delivery reports. For mothers that started breastfeeding, breastfeeding duration was categorized into 3 groups: $<6$ months, $6-12$ months, and $>12$ months. The first category ( $<6$ months) was selected as the reference group based on WHO advice to breastfeed for at least 6 months, and on national figures showing that most children in The Netherlands are breastfed for $<6$ months [World Health Organization, 2002; Peeters et al., 2015]. The third category ( $>12$ months) was based on the minimal duration of breastfeeding, which showed an association with caries in previous research; no information to determine a duration of breastfeeding of $>12$ months was available in our study [Peres et al., 2018]. Duration of exclusively breastfeeding was based on the age when other types of milk and/or foods were introduced in the first 6 months of life according to the questionnaire at 6 months. Since the number of mothers who exclusively breastfed for $\geq 6$ months was very low $(n=32[1 \%])$, exclusively breastfeeding was categorized as: nonexclusive until 4 months of age; and exclusive until 4 months of age; the former applied to infants who received both breast milk and formula or solids during the first 4 months of life, and the latter indicated infants who had been 
Fig. 1. Flowchart showing the selection of

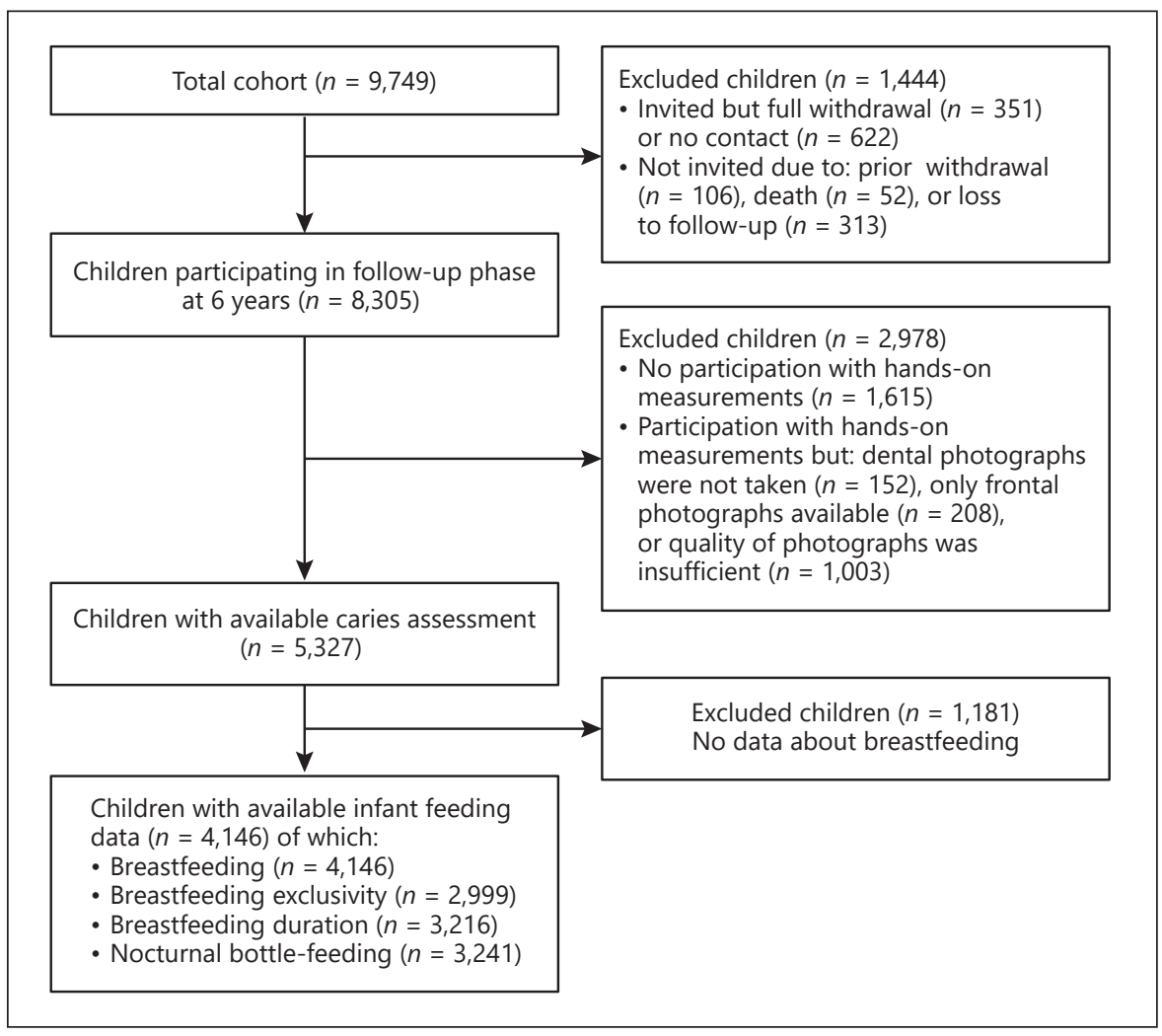
the study population.

breastfed without any other milk, solids, or fluids during the first 4 months of life. Information on the frequency of breastfeeding was obtained when children were 6 and 12 months old, and mothers were asked how often they were breastfeeding their child at those time points. Information about nocturnal bottle-feeding was retrieved at the age of 36 months and mothers had to answer the question: How often has your child received bottle-feeding during the night in the last month (not including tea or water)?

\section{Assessment of Dental Caries}

At the visit to the research center at around the age of 6 months, intraoral photographs were made by trained personnel (Poscam USB intraoral autofocus camera, Digital Leader PointNix, $640 \times$ 480 pixels, minimal scene illumination of 1.4 and $30 \mathrm{~lx}$ ). Before taking the photographs, teeth were brushed and excess saliva was removed with a cotton roll. Approximately 10 photographs were made, capturing the whole dentition, i.e., showing the occlusal, buccal, lingual, and palatal sides of all teeth. All photographs were scored by a single trained dentist (M.E.) using full-screen mode on a computer, and $10 \%$ of the photographs were scored again after at least 6 weeks to calculate intraobserver reliability (Cohen's $\kappa=$ 0.80 ). Approximately $10 \%$ of the photographs were scored by a second trained pediatric dentist, using the same method to calculate interobserver reliability (Cohen's $\kappa=0.76$ ) [Elfrink et al., 2009]. Dental caries was assessed in the primary dentition using the decayed, missing, and filled teeth (dmft) index [World Health Organization, 2013]. Decayed teeth were assessed as lesions extended into dentin (enamel caries was not taken into account). Missing teeth were only assessed when teeth were extracted due to caries, and this was individually judged based on the dental development and caries pattern of the child. Filled teeth were scored if teeth were restored due to caries only by consideration of the size and form of the restoration, and the general status of the child's dentition. The use of intraoral photographs for scoring dental caries per tooth in epidemiological studies showed high sensitivity and specificity ( 85.5 and $83.6 \%$, respectively) compared to the clinical visual tactile inspection made in a previous validation study [Elfrink et al., 2009].

\section{Assessment of Other Variables}

The following variables were considered potential confounders: child's age and gender, family SEP, and ethnic background. Consumption of carbohydrates and sugar-rich products were considered as potential mediating factors.

Indicators of family SEP used in this study were maternal educational level, net household income, marital status, and the employment status of the mother. Educational level was defined according to Statistics Netherlands into 3 categories: "low" (no education, primary education, or secondary phase 1 finished), "middle" (secondary phase 2 finished) and "high" (higher vocational training or a university degree finished) [CBS, 2017]. Net household income per month was dichotomized using the approximate monthly general net labor income during the enrolment period of this cohort as the cut-off point ( $\leq$ EUR 2,200 and $>$ EUR 2,200) [CBS, 2012].

Ethnic background was defined according to the Dutch classification, which is determined by the parents' place of birth [CBS, 2004]. If both parents of the child were born in The Netherlands, 
ethnic background was classified as "Dutch". If one of the parents was born in another country, ethnic background was classified as "non-Dutch".

Dietary intake at 1 year was measured using validated semiquantitative food frequency questionnaires (FFQs) that included 211 items, which were filled out by the caregiver of the child. More details of the development and validation of the FFQ can be found elsewhere [Voortman et al., 2015]. The daily intake of carbohydrate in grams (excluding breastmilk) was calculated with the use of the Dutch Food Composition Table 2006 [Netherlands Nutrition Centre, 2006].

The intake of sugar containing products during childhood was estimated from parental questionnaires when the child was 6 years old. Parents were asked about the frequency of caloric snack consumption (e.g., candies, chocolate bars, or ice-cream) and sugarsweetened beverages during the day (e.g., soft drinks, packaged fruit juices, lemonade, sweetened milk products like chocolate milk, and tea with sugar). For analyses, this variable was dichotomized using 3 sugar-containing products per day as the cut-off point based on previous research [Peres et al., 2017].

\section{Data Analyses}

Because data on infant feeding practices were not complete for all participants, the sample size varied per specific analysis (range 2,999-4,146).

We applied negative binomial hurdle regression models to study the associations between several infant feeding practices and dental caries. Hurdle models allow for a better estimation of an association when the outcome of interest is zero-inflated and overdispersed, such as dmft as a measure of caries [Hofstetter et al., 2016]. A hurdle model consists of 2 parts: a zero-part equal to binomial logistic regression that estimates the odds ratio (OR) of having caries experience compared to children without caries, and a count-part which estimates the contribution of infant feeding practices to the amount of caries experience for those individuals with $\mathrm{dmft} \geq 1$ using rate ratios (RR). Analyses concerning exclusively breastfeeding in the first 4 months and breastfeeding duration were performed in breastfed children only. Three models were built. The first included the age and gender of the child. The second was adjusted for indicators of family SEP (the mother's educational level, the net family household income, the marital status of the mother, and maternal employment status), and ethnic background. In the third model, we adjusted for total intake of carbohydrates in grams per day at 1 year, and intake of sugar-containing products at the age of 6 years as potential mediating factors. No relevant change in effect estimates was observed in the third model, so advanced methods for mediation analyses were not considered further.

Multiple imputation on the study population of 4,146 children was performed to account for information bias associated with missing data in the covariates [Sterne et al., 2009]. Missing values of sociodemographic and lifestyle variables were multiple-imputed by generating 20 independent datasets with the use of chained equations, and the effect estimates for each imputed dataset were pooled and presented in this paper. Imputations were based on all variables in the models, but the main determinants and the outcome were not imputed. Because similar effect estimates were found in the analyses with imputed and unimputed data, the pooled results of the imputed dataset were presented. The statistical analyses were performed using SPSS (IBM Corp. Released
2017. IBM SPSS Statistics for Windows, v25.0. Armonk, NY, USA) and $R$ v3.5.2 ( $R$ Core Team, Vienna, Austria; packages: mice and pscl).

\section{Supplementary Analyses}

Brushing frequency retrieved from a parental questionnaire when children were 3 years old, was calculated for each category of breastfeeding duration (online suppl. Table S1; for all online suppl. material, see www.karger.com/doi/10.1159/000514502). To study the influence of brushing frequency on the association between infant feeding and dental caries, the main models were additionally adjusted for brushing frequency (online suppl. Table S2).

To observe whether the number of breastfeeding moments differed between children with and without caries, the frequency of breastfeeding when children were 12 months old was calculated for caries-free children and caries-active children (online suppl. Table S3).

To observe potential selection bias, a nonresponse analysis was performed to compare all 6-year-old children with and without missing data on infant feeding and caries experience (online suppl. Table S4).

\section{Results}

Children had a mean age (SD) of $6.1(0.4)$ years when dental imaging took place. Of the total study population, $92.4 \%(n=3,831)$ of the children had been breastfed, 847 $(28.2 \%)$ were exclusively breastfed in the first 4 months, $386(12.4 \%)$ were breastfed for $>12$ months, and 409 $(12.6 \%)$ received nocturnal bottle-feeding. The prevalence of caries at the age of 6 years was $27.9 \%(n=1,158)$ with a mean (SD) of 0.97 (2.17), and dental caries was more prevalent in children from families with a low SEP (Table 1).

The results of the negative binomial hurdle models showed that, after adjustment for confounding and potential mediating factors, prolonged duration of breastfeeding (>12 months) was significantly associated with dental caries (model 3: OR 1.35, 95\% CI 1.04-1.74). In addition, of the children with dental caries $(\mathrm{dmft}>0)$, prolonged breastfeeding also significantly increased the number of teeth affected by dental caries by $27 \%$ (Model 3: RR $1.27,95 \%$ CI 1.03-1.56) compared to infants that were breastfed until 6 months. Model 3 also showed a significant association between nocturnal bottle-feeding and dental caries (OR 1.52, 95\% CI 1.20-1.93), but no increase in the number of teeth affected by dental caries among children with $\mathrm{dmft}>0$ (RR 1.02 , 95\% CI 0.84 1.24). Furthermore, no association was observed between having ever been breastfed or exclusively breastfed in the first 4 months and dental caries at the age of 6 years. The 
Table 1. Characteristics of the study population

$\begin{array}{lll}\begin{array}{l}\text { Total } \\ (n=4,146)\end{array} & \mathrm{dmft}=0 & \mathrm{dmft}>0 \\ (n=2,988,72.1 \%) & (n=1,158,27.9 \%)\end{array}$

Child's gender

Boys

Girls

Child's age, years

$2,049(49.4)$

$1,467(71.6)$

$582(28.4)$

$2,097(50.6)$

$1,521(72.5)$

$576(27.5)$

$6.1 \pm 0.4$

$6.0 \pm 0.3$

$6.2 \pm 0.5^{*}$

Ever breastfed

Yes

No

$3,831(92.4)$

$2,764(72.1)$

$315(7.6)$

$224(71.1)$

$1,067(27.9)$

$91(28.9)$

Exclusively breastfed in the first 4 months

Yes

$847(28.2)$

$655(77.3)$

$1,581(73.5)$

$752(65.6)$

$192(22.7)^{*}$

$2,152(71.8)$

$752(65.6)$

$571(26.5)$

Missing data

1,147 (27.7)

$1,494(75.5)$

$395(34.4)$

$1,980(61.6)$

$638(75.1)$

$486(24.5)^{*}$

$850(26.4)$

$261(67.6)$

$212(24.9)$

$386(12.0)$

$595(64.0)$

$125(32.4)$

Missing d

$930(22.4)$

$2,188(77.3)$

$335(37.0)$

Nocturnal bottle-feeding

Never

$2,832(87.4)$

$2,188(77.3)$
$254(62.1)$

$644(22.7)^{*}$

409 (12.6)

$546(60.3)$

$155(37.9)$

Missing data

$905(21.8)$

379 (51.6)

359 (39.7)

$734(17.7)$

$886(70.0)$

$355(48.4)^{*}$

$1,264(30.5)$

$1,723(80.2)$

$378(30.0)$

Middle

$2,148(51.8)$

$1,010(61.0)$

425 (19.8)

Net income per month ${ }^{\mathrm{a}}$

Low (< EUR 2,200)

High (> EUR 2,200)

$1,655(39.9)$

$1,978(79.4)$

$645(39.0)^{*}$

$2,491(60.1)$

$2,675(72.3)$

$513(20.6)$

3,701 (89.3)

$313(70.3)$

$1,026(27.7)$

No partner

$445(10.7)$

$2,442(76.0)$

$132(29.7)$

$3,212(77.5)$

$546(58.4)$

$770(24.0)^{*}$

$388(41.5)$

Unemployed

$934(22.5)$

2,442 (58.9)

$1,933(79.2)$

$509(20.8)^{*}$

Dutch

$1,704(41.1)$

1,055 (61.9)

$649(38.1)$

Total carbohydrate intake (without breast milk) at 1 year, $\mathrm{g} / \mathrm{day}^{\mathrm{a}}$

Intake of sugar-containing products at 6 years $^{\mathrm{a}}$ Low ( $\leq 2$ times per day)

High ( $>2$ times per day)

$187.3 \pm 58.4$

$187.6 \pm 56.6$

$186.5 \pm 62.7$

$1,407(33.9) \quad 1,033(73.4)$

$374(26.6)$

$2,739(66.1) \quad 1,955(71.4)$

784 (28.6)

$0.97 \pm 2.17$

0-19

$3.46 \pm 2.87$

Range

1-19

Values are expressed as $\mathrm{n}(\%)$ or mean $\pm \mathrm{SD}$, unless otherwise indicated. * Statistical significance difference $(p<0.05)$ in mean or distribution of the variable compared to children without caries $(\mathrm{dmft}=0)$, estimated using independent $t$ tests and $\chi^{2}$ tests for continuous and categorical variables, respectively.

a Based on imputed data. Number of missing values per variable: 273 for educational level, 818 for income, 283 for marital status, 767 for employment status, 62 for ethnic background, 1,745 for total carbohydrate intake and 617 for intake of sugar products. 
Table 2. Associations between infant feeding practices and dental caries ${ }^{\mathrm{a}}$

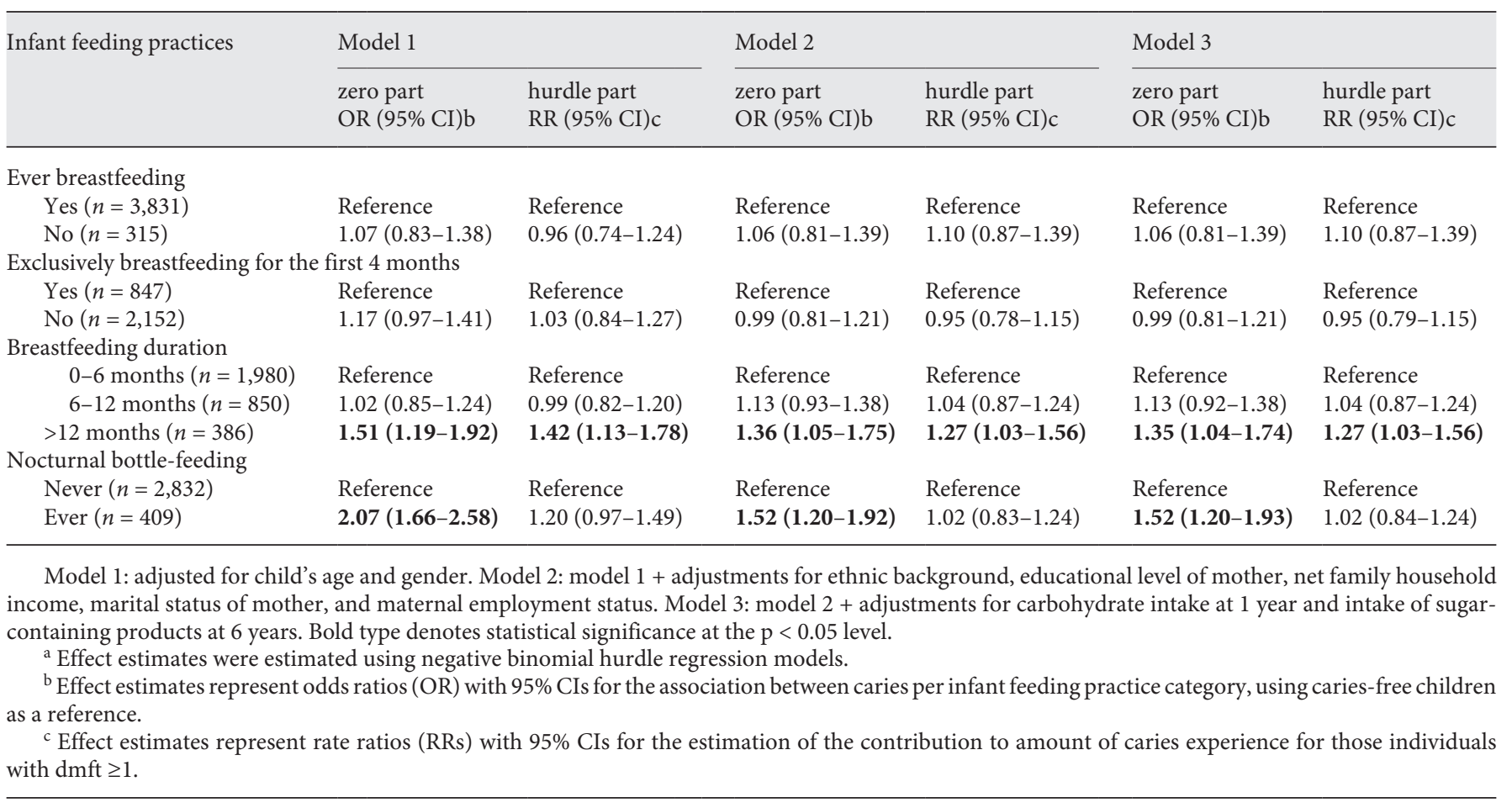

addition of sugar intake as a potential mediating factor in model 3 did not show any relevant changes in effect estimates compared to model 2 (Table 2).

\section{Discussion}

The results of our study on a Dutch cohort show that breastfeeding for $>12$ months is associated with an increased risk of having dental caries and an increase in the total $\mathrm{dmft}$ count at the age of 6 years. Nocturnal bottlefeeding in infancy is also associated with a risk of having dental caries at the age of 6 years. Both associations remained after adjustments for indicators of family SEP, ethnic background, and intake of carbohydrates and sugar-rich products.

A number of studies have investigated the association between breastfeeding and caries. However, comparison is not always straightforward because of differences in the categorization of breastfeeding duration and the study designs. The studies that have shown an association between prolonged breastfeeding duration and caries (as in our study) included at least a comparison of breastfeeding duration categories of shorter versus longer than 12,18 , or 24 months [Peres et al., 2018]. As summarized in 2 sys- tematic reviews, previous studies, overall, indicated that breastfeeding for $>12$ months can increase the risk of caries, in line with our results [Tham et al., 2015; Cui et al., 2017]. In contrast to our findings, the results of 2 other systematic reviews showed that any breastfeeding, in general, is protective against dental caries compared to children who never received breast milk [Avila et al., 2015; Cui et al., 2017]. However, most of the individual studies included in these reviews had cross-sectional study designs. Our study had a prospective cohort design, which has a higher potential to distinguish between cause and effect because of the chronological order of exposure and outcome measurements. Only 3 prospective cohort studies included in the abovementioned systematic reviews considered prolonged breastfeeding of at least 12 months as a reference category and took into account relevant socioeconomic indicators and other nutritional behaviors. Two of the studies found a significant association between being breastfed for $>12$ or 24 months and dental caries [Feldens et al., 2010; Chaffee et al., 2014], and another found a nonsignificant but increased odds of early childhood caries with a breastfeeding duration of $>18$ months [Tanaka et al., 2013]. Four recent prospective cohort studies (i.e., not included in these systematic reviews) investigated the association between breastfeeding 
and dental caries in children. Two confirmed an association between prolonged breastfeeding ( $>24$ months) and caries [Peres et al., 2017; Wong et al., 2017] and 2 confirmed a nonsignificant trend between breastfeeding for $>12$ or $>18$ months and caries [Nirunsittirat et al., 2016; Devenish et al., 2020]. All of these recent studies have considered indicators of SEP which are known to be strongly related to breastfeeding practices and dental caries [Peres et al., 2017; Wong et al., 2017; Devenish et al., 2020], and 2 of the studies considered sugar intake as a possible confounding or mediating factor [Peres et al., 2017; Devenish et al., 2020].

A review on breastfeeding-related health consequences that appeared in the Lancet proposed that a lack of proper oral hygiene after breastfeeding may explain the higher risk of caries found in children with prolonged breastfeeding [Victora et al., 2016]. However, in our study, brushing frequency was not related to breastfeeding behaviors (online suppl. Table S1), and did not explain the observed relationship between prolonged breastfeeding and caries (online suppl. Table S2). We therefore did not adjust for brushing frequency as a potential confounder, which is also supported by other literature [Peres et al., 2018]. Breastfeeding in itself and of prolonged duration, has been shown to be beneficial for many health outcomes, such as overweight, diabetes, and infections [Victora et al., 2016]. We do not want to undermine the current advice from the WHO [2002] about breastfeeding when discussing the underlying mechanism between prolonged breastfeeding and dental caries. Previous authors have concluded that the risk of caries further increases when prolonged breastfeeding involves nocturnal feeds [Tham et al., 2015]. Furthermore, 2 previous studies showed that prolonged breastfeeding was more strongly associated with dental caries when breastfeeding was frequent, compared to infrequent prolonged breastfeeding, even after adjustments for other dietary practices [Feldens et al., 2010; Chaffee et al., 2014]. Our adjustments for total daily carbohydrate intake at the age of 12 months had no relevant effect on the overall findings. In addition, the proportion of children with dental caries was clearly higher among infants who had been exposed to increased breastfeeding frequency at the age of 12 months (online suppl. Table S3), which points towards the theory that increased feeding frequency might be the major explanation for the observed association between prolonged breastfeeding and dental caries. As advised by the WHO [2002], from 6 months onwards, infants are introduced to complementary feeding next to breastfeeding. At the same time, the first deciduous teeth erupt, i.e.,

Breastfeeding and Childhood Dental Caries when a child is approximately $8-12$ months of age. Since human milk has cariogenic characteristics, given the high lactose concentration [Bowen and Lawrence, 2005], a higher frequency of any infant feeding may increase the risk of dental caries (also shown in a previous study [Feldens et al., 2018]). The theory of feeding frequency and the risk of caries is based on the imbalance between demineralization (loss of minerals from enamel caused by low $\mathrm{pH}$ ) and remineralization (repair of enamel by minerals from the saliva or supplemented fluoride). Reduction of carbohydrate intake frequency is one of the most important methods which decreases demineralization and thereby promotes remineralization [Gustafsson et al., 1954; Featherstone, 2004]. Therefore, the Dutch Association for Oral Health advises a maximum of 7 eating and drinking moments per day. Unfortunately, no guidance concerning breastfeeding is included in this recommendation [Ivoren Kruis, 2011]. Our results also confirm that nocturnal bottle-feeding is related to an increased caries risk. This relationship is established earlier and is probably related to the content of the bottle. Also, during the night, the salivary flow is less, leading to decreased remineralization which increases the risk of developing caries lesions over time [Shelton et al., 1977; Reisine and Psoter, 2001].

The results of our study must be seen in the light of some limitations. Unfortunately, we did not have information about nocturnal breastfeeding, breastfeeding on demand, or feeding frequency at the age of 12 months, and we could only speculate about the potential mechanism underlying the observed association of prolonged breastfeeding with dental caries. Furthermore, dental caries was assessed using intraoral photographs. Since it is more difficult to differentiate between stages of caries development using intraoral photographs, we assessed dentin caries only and enamel caries was not evaluated. We do not expect that this potential misclassification and underestimation of caries is related to feeding patterns; nevertheless, it is a nondifferential error which might have caused an underestimation of the effect estimates. In addition, caries was assessed only once, and we were not able to study the timing of dental caries in relation to infant feeding practices. However, $\mathrm{dmft}$ is a cumulative measure indicating caries activity during the whole of childhood and includes both treated and untreated caries in the primary dentition. Also, at the age of 6 years, some children might have exfoliated some primary teeth. Some children might be wrongly classified as caries-free, but in fact have had caries in the primary dentition. We partly took this into account by adjusting for age at the caries 
assessment in our main analyses. Nevertheless, this limitation could have led to a nondifferential information bias, which could have diluted the effect estimates. Selection bias due to differential participation could also have played a role, since participants with missing data on infant feeding or dental caries had higher levels of dental caries and a lower family SEP (online suppl. Table S4). This could have induced either under- or overestimation of the associations. Moreover, data about oral hygiene around the period of eruption of the primary teeth was not available in our study. In The Netherlands, children might be exposed to fluoride only through toothpaste since all toothpastes contain fluoride. If the children that breastfed for $>12$ months brushed their teeth less than those who did not breastfeed for $>12$ months, this could have led to a nonrandom error which could have overestimated the results of this study. Lastly, nocturnal bottlefeeding was defined via a questionnaire when children were 3 years old as: "ever" or "never in the previous month." This is likely to have underestimated the true prevalence of nocturnal bottle-feeding. Therefore, the effect estimate of the association between nocturnal bottlefeeding and dental caries might have been biased towards the null, and explain why no association was found between nocturnal bottle-feeding and increased risk in total $\mathrm{dmft}$ count in children with caries.

The major strengths of the study are the large multiethnic study population based on a longitudinal cohort and the use of hurdle regression models. Also, this is one of the few studies on this topic in a European context. We showed that, in a western European country, with infant feeding practices and oral health care different from in non-European countries, prolonged breastfeeding is (still) associated with caries. We were able to adjust for a diverse set of indicators for SEP and dietary factors, which makes the association between breastfeeding duration and caries from this study even more robust.

\section{Conclusion}

We have shown that prolonged breastfeeding as well as bottle-feeding during the night are associated with an increased risk of childhood dental caries. Our findings confirm the results of earlier studies in other countries and add that the associations are independent of SEP and sugar intake, and also exist in a European context that is without water fluoridation. Although future studies are encouraged that will study the potential mechanism between prolonged breastfeeding and dental caries in more detail, the evidence so far clearly shows a higher risk of dental caries in children being breastfed for $>12$ months. Breastfeeding in itself, and of prolonged duration, should not be discouraged since it has been shown to be beneficial for many health outcomes [Victora et al., 2016]. However, healthcare professionals should use the current recommendations on breastfeeding, oral hygiene, and feeding frequency to advise parents about the benefits of breastfeeding and proper oral hygiene, but at the same time also about the risk of increased frequency of any feeding, including breastfeeding, after tooth eruption.

\section{Acknowledgments}

The Generation R Study is being conducted by the Erasmus Medical Centre in close collaboration with the School of Law and the Faculty of Social Sciences of the Erasmus University, Rotterdam; the Municipal Health Service, Rotterdam area; the Rotterdam Homecare Foundation; and the Stichting Trombosedienst en Artsenlaboratorium Rijnmond, Rotterdam, the Netherlands. The authors gratefully acknowledge the contributions of the general practitioners, hospitals, midwives, and the pharmacies in Rotterdam.

\section{Statement of Ethics}

The study was approved by the Medical Ethical Committee of Erasmus Medical Centre, Rotterdam, the Netherlands (MEC 198.782/2001/31) and conducted according to the World Medical Association Declaration of Helsinki. All examinations, interviews and questionnaires were carried out after obtaining written informed consent of parent(s) or legal guardian(s) of the participants.

\section{Conflict of Interest Statement}

The authors declare no potential conflicts of interest with respect to the authorship and/or publication of this article.

\section{Funding Sources}

This work was supported by financial support from the Erasmus Medical Centre, Erasmus University Rotterdam, The Netherlands Organization for Health Research and Development, The Netherlands Organization for Scientific Research, the Ministry of Health Welfare and Sport, the Ministry of Youth and Families, and the European Research Council. The dental caries assessment of the study was financially supported by an unrestricted grant of GABA International, Therwil, Switzerland. 


\section{Author Contributions}

A.W.M.L. contributed to the conception and design of the study, carried out the statistical analyses, interpreted the data, drafted the initial manuscript, and revised the manuscript; T.V. contributed to the acquisition of infant feeding data and reviewed and revised the manuscript; M.E.C.E contributed to the acquisi- tion of dental data and critically reviewed and revised the manuscript; E.B.W. contributed to the conception and design of the study and critically reviewed and revised the manuscript; L.K. contributed to the conception and design of the study, interpreted the data, and critically reviewed and revised the manuscript; All authors approved the final manuscript as submitted and agree to be accountable for all aspects of the work.

\section{References}

Avila WM, Pordeus IA, Paiva SM, Martins CC Breast and Bottle Feeding as Risk Factors for Dental Caries: A Systematic Review and MetaAnalysis.PLoSOne.2015Nov;10(11):e0142922.

Bowen WH, Lawrence RA. Comparison of the cariogenicity of cola, honey, cow milk, human milk, and sucrose. Pediatrics. 2005 Oct;116(4) 921-6.

CBS. Immigrants in the Netherlands 2004 (Allochtonen in Nederland 2004). Den Haag/Heerlen: Statistics Netherlands. Centraal Bureau voor de Statistiek; 2004

CBS. Income, welfare and spending of households and individuals. Den Haag/Heerlen: Statistics Netherlands (Centraal Bureau voor de Statistiek); 2012

CBS. The Dutch Standard Classification of Education (Standaard onderwijsindeling 2016). Den Haag/Heerlen: Statistics Netherlands. Centraa Bureau voor de Statistiek; 2017.

Chaffee BW, Feldens CA, Vítolo MR. Association of long-duration breastfeeding and dental caries estimated with marginal structural models. Ann Epidemiol. 2014 Jun;24(6):448-54

Cui L, Li X, Tian Y, Bao J, Wang L, Xu D, et al. Breastfeeding and early childhood caries: a meta-analysis of observational studies. Asia Pac J Clin Nutr. 2017;26(5):867-80.

Devenish G, Mukhtar A, Begley A, Spencer AJ, Thomson WM, Ha D, et al. Early childhood feeding practices and dental caries among Australian preschoolers. Am J Clin Nutr. 2020 Apr; 111(4):821-8.

Durmus B, van Rossem L, Duijts L, Arends LR, Raat $\mathrm{H}$, Moll HA, et al. Breast-feeding and growth in children until the age of 3 years: the Generation R Study. Br J Nutr. 2011 Jun;105(11):1704-11.

Elfrink ME, Veerkamp JS, Aartman IH, Moll HA Ten Cate JM. Validity of scoring caries and primary molar hypomineralization $(\mathrm{DMH})$ on intraoral photographs. Eur Arch Paediatr Dent. 2009 Nov;10(Suppl 1):5-10.

Featherstone JDB. The continuum of dental caries-evidence for a dynamic disease process. J Dent Res. 2004;83 Spec No C(1 suppl):C39-42.

Feldens CA, Giugliani ER, Vigo Á, Vítolo MR. Early feeding practices and severe early childhood caries in four-year-old children from southern Brazil: a birth cohort study. Caries Res. 2010; 44(5):445-52.

Feldens CA, Rodrigues PH, de Anastácio G, Vítolo MR, Chaffee BW. Feeding frequency in infancy and dental caries in childhood: a prospective cohort study. Int Dent J. 2018 Apr;68(2):11321.

Gustafsson BE, Quensel CE, Lanke LS, Lundqvist C, Grahnen H, Bonow BE, et al. The Vipeholm dental caries study; the effect of different levels of carbohydrate intake on caries activity in 436 individuals observed for five years. Acta Odontol Scand. 1954 Sep;11(3-4):232-64.

Ha DH, Spencer AJ, Peres KG, Rugg-Gunn AJ, Scott JA, Do LG. Fluoridated Water Modifies the Effect of Breastfeeding on Dental Caries. J Dent Res. 2019 Jul;98(7):755-62.

Hofstetter H, Dusseldorp E, Zeileis A, Schuller AA. Modeling Caries Experience: Advantages of the Use of the Hurdle Model. Caries Res. 2016; 50(6):517-26

Ivoren Kruis. [Internet] Caries Prevention Advice. Practical guideline for professionals about preventive oral health care [cited Nov 2011]. Dutch. Available from: http://www.dentagon. nl/wp-content/uploads/2012/02/AdviesCari\%C3\%ABspreventie-.pdf

Jaddoe VW, Mackenbach JP, Moll HA, Steegers EA, Tiemeier H, Verhulst FC, et al. The Generation R Study: design and cohort profile. Eur J Epidemiol. 2006;21(6):475-84.

Kassebaum NJ, Bernabé E, Dahiya M, Bhandari B, Murray CJ, Marcenes W. Global burden of untreated caries: a systematic review and metaregression. J Dent Res. 2015 May;94(5): 650-8.

Kooijman MN, Kruithof CJ, van Duijn CM, Duijts $\mathrm{L}$, Franco $\mathrm{OH}$, van IJzendoorn $\mathrm{MH}$, et al. The Generation R Study: design and cohort update 2017. Eur J Epidemiol. 2016 Dec;31(12):124364.

Lagerweij MD, van Loveren C. Declining Caries Trends: Are We Satisfied? Curr Oral Health Rep. 2015;2(4):212-7.

Netherlands Nutrition Centre. Dutch Food Composition Database 2006 (NEVO-tabel 2006). Den Haag: Netherlands Nutrition Centre (Voedingscentrum); 2006.

Nirunsittirat A, Pitiphat W, McKinney CM, DeRouen TA, Chansamak N, Angwaravong O, et al. Breastfeeding Duration and Childhood Caries: A Cohort Study. Caries Res. 2016;50(5):498507.

Peeters D, Lanting CI, Van Wouwe JP. Peiling melkvoeding van zuigelingen 2015. Leiden, the Netherlands: TNO; 2015.

Peres KG, Chaffee BW, Feldens CA, Flores-Mir C, Moynihan P, Rugg-Gunn A. Breastfeeding and
Oral Health: Evidence and Methodological Challenges. J Dent Res. 2018 Mar;97(3):251-8.

Peres KG, Nascimento GG, Peres MA, Mittinty MN, Demarco FF, Santos IS, et al. Impact of Prolonged Breastfeeding on Dental Caries: A Population-Based Birth Cohort Study. Pediatrics. 2017 Jul;140(1):e20162943.

Reisine ST, Psoter W. Socioeconomic status and selected behavioral determinants as risk factors for dental caries. J Dent Educ. 2001 Oct;65(10): 1009-16.

Selwitz RH, Ismail AI, Pitts NB. Dental caries. Lancet. 2007 Jan;369(9555):51-9.

Shelton PG, Berkowitz RJ, Forrester DJ. Nursing bottle caries. Pediatrics. 1977 May;59(5):777-8.

Sterne JA, White IR, Carlin JB, Spratt M, Royston $\mathrm{P}$, Kenward MG, et al. Multiple imputation for missing data in epidemiological and clinical research: potential and pitfalls. BMJ. 2009 Jun; 338:b2393.

Tanaka K, Miyake Y, Sasaki S, Hirota Y. Infant feeding practices and risk of dental caries in Japan: the Osaka Maternal and Child Health Study. Pediatr Dent. 2013 May-Jun;35(3):267-71.

Tham R, Bowatte G, Dharmage SC, Tan DJ, Lau MX, Dai X, et al. Breastfeeding and the risk of dental caries: a systematic review and meta-analysis. Acta Paediatr. 2015 Dec;104(467):62-84.

Victora CG, Bahl R, Barros AJ, França GV, Horton S, Krasevec J, et al.; Lancet Breastfeeding Series Group. Breastfeeding in the 21st century: epidemiology, mechanisms, and lifelong effect. Lancet. 2016 Jan;387(10017):475-90.

Voortman T, Kiefte-de Jong JC, Geelen A, Villamor E, Moll HA, de Jongste JC, et al. The development of a diet quality score for preschool children and its validation and determinants in the Generation R Study. J Nutr. 2015 Feb;145(2): 306-14.

Watt R, Sheiham A. Inequalities in oral health: a review of the evidence and recommendations for action. Br Dent J. 1999 Jul 10;187(1):6-12.

Wong PD, Birken CS, Parkin PC, Venu I, Chen Y, Schroth RJ, et al. Total Breast-Feeding Duration and Dental Caries in Healthy Urban Children. Acad Pediatr. 2017 Apr;17(3):310-5.

World Health Organization. Infant and young child nutrition. Global strategy on infant and young child feeding. Fifty-fifth World Health Assembly; 2002. p. 55.

World Health Organization. Oral health surveys: basic methods. World Health Organization; 2013. 